\title{
Research on robot indoor localization method based on wireless sensor network
}

\author{
Wengpeng WANG ${ }^{1, a}$, Xin WANG ${ }^{2}$ \\ ${ }^{1}$ School of Information and Electronics, Beijing Institute of Technology \\ ${ }^{2}$ Ministry of Education Virtual Simulation Experiment Teaching Centers \\ aemail: wangwp1993@126.com
}

Keywords: robot indoor location; RSSI; trilateration centroid localization algorithm; weighted centroid localization algorithm

\begin{abstract}
This paper use RSSI wireless sensor network (WSN) to research robot indoor localization method. Location simulation analysis, which is processed by Matlab software, is used to adapt trilateration centroid localization algorithm (TCLA) and weighted centroid localization algorithm (WCLA). To verify the algorithm positioning accuracy and feasibility, location test system is built in the laboratory. The experiment results indicated that two algorithms both were simple and had high accuracy. They could meet requirement of motive robot or car indoor location. Compared with the two algorithms, TCLA had high location accuracy when reference points less than or equal three. But if reference points are more than three points, WCLA would have a better result. When reference points more than or equal eight, location precision error should be less than 0.0125 meter and location error less than $0.4 \%$.
\end{abstract}

\section{Introduction}

Self localization is one of the necessary functions of autonomous mobile robot. By detecting the internal state or percept the external environment, robot will estimate its own location. At present, to get robot location , application of global positioning system (GPS), Wi-Fi, RFID, Bluetooth, wireless sensor devices has made some satisfied research results. But limit to the application occasions, actual working conditions, signal characteristics and other factors, most mobile robot localization use information acquired by airborne sensors to achieve positioning. At present, indoor localization method for target point mainly has: the signal fingerprint recognition ${ }^{[1]}$, TOA or TDOA by the use of UWB technology, localization method in wireless sensor networks ${ }^{[2]}$, integrated navigation ${ }^{[3]}$, data fusion ${ }^{[4]}$ and research on indoor and outdoor seamless conversion .Current localization algorithms is mainly divided into two categories: range based algorithm (range-based) ${ }^{[5]}$ and range free algorithm (range-free) ${ }^{[6]}$. Among all of algorithms, trilateration algorithm is a widely used one and also easy to conduct.

In this paper, we take WSN technology into robot indoor dynamic tracking system. Base on RSSI wireless location positioning theory. Using software analysis to compare location accuracy and applicable conditions of trilateration algorithm and WCLA. By evaluating two algorithms, advantages of each algorithm is elaborated and analysis. After that, laboratory indoor location testing system is established to verify the simulation results of two algorithms.

\section{Location algorithms}

In wireless sensor networks, ranging methods based on ranging algorithms mainly include Received Signal Strength Indication (RSSI), Time of Arrival (TOA), Angel of Arrival (AOA) and Time Difference of Arrival (TDOA). Among them, RSSI is the most wildly used one. The" Received Signal Strength Indication-Distance" model is simple and feasible. This paper will use this method.

\subsection{Weighted centroid location model}

Formula (3) is the mathematical expression of RSSI model. A node transmits a signal while 
another one receives. The distance between these two nodes can be calculated by measuring the received signal strength according to formula (3).

This paper Locate the target node on the basis of using trilateration. Trilateration is a localization algorithm based on distance. Provided unknown node $\mathrm{D}$ coordinates (X, Y).A, B, and C point coordinates are separately (X1, Y1), (X2, Y2) and (X3, Y3).Their distance to D respectively are d1, $\mathrm{d} 2$, d3.Then get the following equation set:

$$
\left\{\begin{array}{l}
\left(X-X_{1}\right)^{2}+\left(Y-Y_{1}\right)^{2}=d_{1}^{2} \\
\left(X-X_{2}\right)^{2}+\left(Y-Y_{2}\right)^{2}=d_{2}{ }^{2} \\
\left(X-X_{3}\right)^{2}+\left(Y-Y_{3}\right)^{2}=d_{3}{ }^{2}
\end{array}\right.
$$

Solve the equations, then obtain the coordinates of the target node. The principle can be shown as follow figure.

\subsection{TCLA and WCLA}

Due to the measurement error in actual situation, trilateration can't get real solutions in actual use.

(1) Respectively denote the three circles A, B and C.Get the intersection of A and B, B and C, C and $\mathrm{A}$ as $\left(\mathrm{X}^{\prime}{ }_{1}, \mathrm{Y}^{\prime}{ }_{1}\right),\left(\mathrm{X}_{2}{ }_{2}, \mathrm{Y}^{\prime}{ }_{2}\right)$ and $\left(\mathrm{X}^{\prime}{ }_{3}, \mathrm{Y}^{\prime}{ }_{3}\right)$. Get the intersection coordinate which is the closest to the third circle center. According to weighted centroid , estimate the coordinate of the target node as:

$$
(X, \quad Y)=\left(\frac{X_{1}^{\prime}+X_{2}^{\prime}+X_{3}^{\prime}}{3}, \frac{Y_{1}^{\prime}+Y_{2}^{\prime}+Y_{3}^{\prime}}{3}\right)
$$

(2)In a wireless network, each node under test receives data sent from reference nodes around. The nodes calculate the distance based on the received RSSI value. With reference nodes whose signal strength is within a certain threshold range, weighted centroid formula can calculate its coordinates.

$$
\left\{\begin{array}{l}
X=\frac{\sum \omega_{i} X_{i}}{\sum \omega_{i}} \\
Y=\frac{\sum \omega_{i} y_{i}}{\sum \omega_{i}}
\end{array}\right.
$$

$\omega_{\mathrm{i}}$ is weight.

Under indoor conditions, the distance between the reference node and the node to be measured won't be large. Get a polygonal region of some reference nodes. We can calculate the centroid coordinates according to estimated distance value between each reference node and node to be measured. During calculating, introduce a weighting factor $\omega_{\mathrm{i}}$. $\omega_{\mathrm{i}}$ will decide the degree of influence reference node affects the weighted centroid coordinate. Calculated according to equation (6). Weighting factor:

$$
\omega=\frac{1}{d}
$$

$d$ is the estimated distance value from the reference node to the node under test.

\section{Software simulation}

In order to test the accuracy of the measurement algorithm, we use Matlab 7.10 as a simulation tool for the research simulation test. Specific steps are as follows: Build a $20 \mathrm{~m} * 10 \mathrm{~m} * 1 \mathrm{~m}$ space coordinate system. Use Matlab software with TCLA and WCLA for simulation test. Assess wireless sensor network node localization algorithm indexes such as node location accuracy, error analysis and some other aspects.

\subsection{TCLA simulation}

TCLA needs three reference nodes. The target nodes are in the triangle area consists of reference nodes. Therefore, select three random reference nodes in Matlab. Generate a random target node in the reference nodes. Take RSSI ranging error into consideration. 


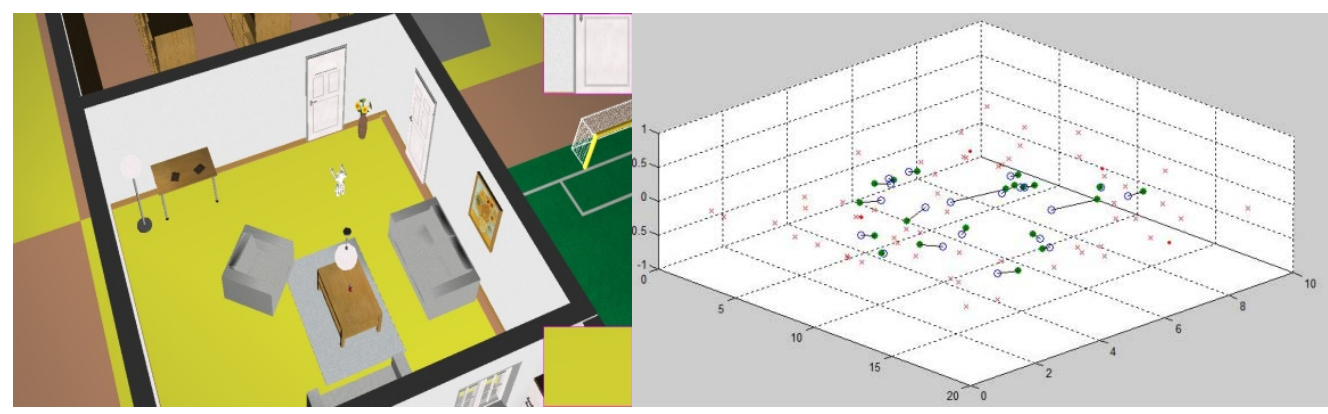

(a)Simulation test virtual scene

(b) Simulation location results

Fig.3 Software virtual location simulation results

Figure 3 (a) shows location algorithm results in the simulation indoor scenes. In figure 3 (b), hollow circle represents the actual coordinates of the target node, while solid circle represents the location coordinates of the target node. $\mathrm{X}$ represents the reference node. The line length is the mean value of location absolute error,represented by E.E is distance mean value between location coordinates and actual coordinates.Cycle program conduct location by increasing target node.Calculate TCLA error value.Error of ten times cycles as shown in table 01.

\subsection{WCLA simulation}

Weighted centroid method and TCLA is slightly different. TCLA only requires three reference nodes, while weighted centroid method can select the number of reference nodes according to the actual situation. The difference number of reference nodes will affect location accuracy. It should be taken into consideration. Figure. 4 respectively shows 3, 8, 14 reference nodes simulation error after 100 times simulation. When the reference nodes number is three, the $E=1.02 \mathrm{~m}$, the relative error is $4.6 \%$. When the reference nodes number is 8 , the $\mathrm{E}=0.52 \mathrm{~m}$, the relative error is $2.3 \%$. When the reference nodes number is 14 , the $\mathrm{E}=0.42$, the relative error is $1.9 \%$.

\subsection{Comparison}

Tabel 1TCLA simulation results (Unit: $\mathrm{m}$ )

\begin{tabular}{|c|c|c|c|c|}
\hline & $\begin{array}{l}\text { Three reference } \\
\text { nodes } \\
\text { TCLA }\end{array}$ & $\begin{array}{l}\text { Three reference } \\
\text { nodes } \\
\text { WCLA }\end{array}$ & $\begin{array}{l}\text { Eight reference } \\
\text { nodes } \\
\text { WCLA }\end{array}$ \\
\hline \multicolumn{2}{|c|}{ Location error } & $0.188 \%$ & $0.200 \%$ & $0.118 \%$ \\
\hline \multirow{4}{*}{$\begin{array}{c}\text { Error } \\
\text { Analysis }\end{array}$} & $\begin{array}{l}\text { Maximum error } \\
\text { distance }\end{array}$ & 3.227 & 2.237 & 1.395 \\
\hline & $\begin{array}{c}\text { Minimum } \\
\text { distance error }\end{array}$ & 0.040 & 0.108 & 0.024 \\
\hline & $\begin{array}{l}\text { Average error } \\
\text { distance }\end{array}$ & 0.106 & 1.004 & 0.069 \\
\hline & $\begin{array}{c}\text { Standard } \\
\text { deviation of error }\end{array}$ & 0.674 & 0.466 & 0.285 \\
\hline
\end{tabular}

The experiment data depicts when reference nodes are of a small number, the weighted centroid error is larger. Increasing the reference nodes number can reduce errors. 2.1 can tell that the trilateral centroid relative error is $4.1 \%$.Compare to the weighted centroid, when the number of reference nodes is three, the relative error of the WCLA relative error is larger than triangular centroid algorithm. When increasing the number of reference nodes, weighted centroid error significantly reduced. The figure also shows that the trilateral centroid algorithm error has a poor stability. Respectively stimulate with trilateral weighted algorithm and centroid centroid algorithm 100 times. Compare the WCLA error when the reference nodes number is different. 


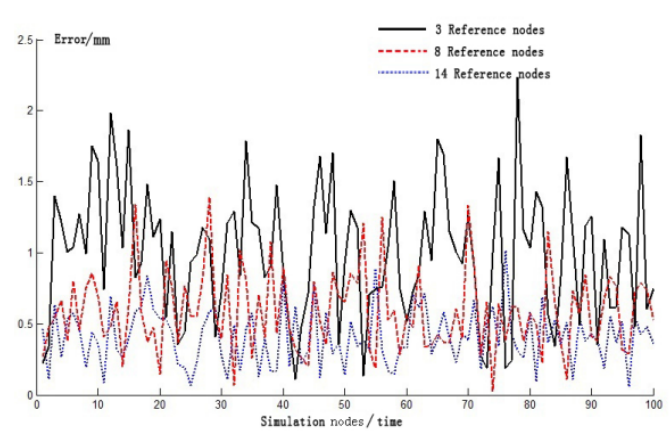

Fig.4 WCLA conditions results

It concludes that in order to reduce error and make location more accurate in real indoor applications, this article tends to choose the WCLA. But the accuracy of the WCLA is affected largely by sensor density and number. Therefore, we should try to increase the number of reference nodes in wireless sensor networks and make reference node location lay out rational.Then we can achieve the desired accuracy.

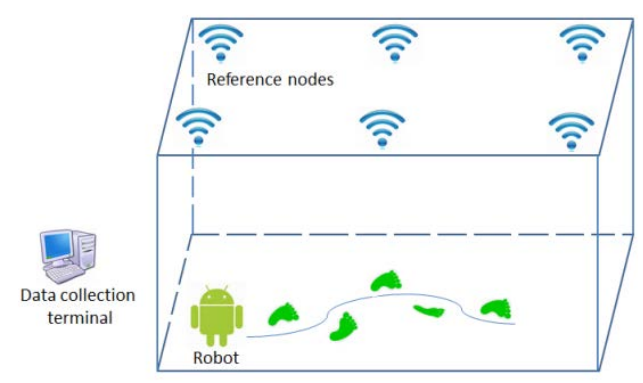

Fig.5 Indoor location system constructure

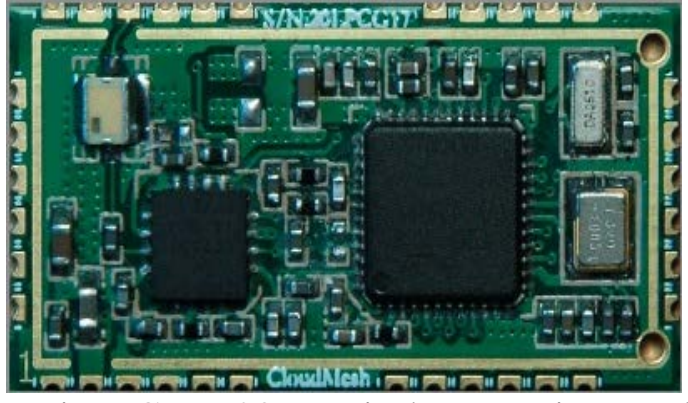

Fig.6 CMT-20LP wireless ranging module

\section{Indoor test experiment}

After determining the location algorithm, we compare the advantages and disadvantages of the two algorithms through experiments. We already conduct simulation tests by using Matlab 7.10. Next, use distance measuring sensors to do indoor positioning experiment in the laboratory environment. Compare and verify with each other by simulation tests.

\subsection{Experiments}

Although WCLA obtain a more ideal positioning accuracy in simulation experiments, the actual test conditions is more complex than the simulation. Measurement data is often affected by vibration of measured target, electromagnetic interference, obstructions and other uncertain factors. So actual indoor experiment is necessary. This paper use CMT-20LP wireless ranging module.CMT-20LP wireless module works in $2.4 \mathrm{GHz}$ ISM band. It can transmit data very fast (up to 2Mbps) and range precisely (outdoor range accuracy up to $1 \mathrm{~m}$ ). The ranging and data communications can be done simultaneously.CMT-20LP module uses linear frequency modulation mode(CSS).Signal bandwidth are $22 \mathrm{MHz}$ and $80 \mathrm{MHz}$. The main chip is wireless transceiver chip nanoLOC TRX Transceiver (NA5TR1) accorded with IEEE802.15.4a standard. It add power amplifier to output and low noise

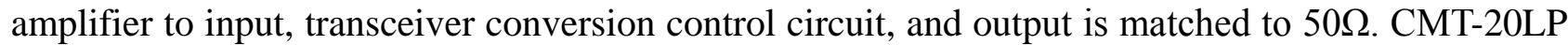
backwards compatible nanoPAN 5375 module which can also directly substitute.

\subsection{Experiment results and analysis}

In "Indoor experiment", install the robot working indoor location trajectory system in a 40m long and 30m wide room to do the verification experiment. The reference node on the top of room obtain distance data of the moving robot and send it to location data collection terminal by using ZigBee wireless communication network .Use WCLA to process collected signals.

Compare the test results with simulation experimental results, the data consistency in location error and error coverage is satisfied. In error analysis results, the error standard deviation of the two algorithms increases significantly. This mainly due to the probability of being blocked and peripheral 
electrical equipment electromagnetic interference increases in the actual indoor test environment. The reliability and consistency of test results decline, which affects the accuracy of localization. But even so, due to the test results, when using WCLA to locate, indexes are still significantly better than TCLA. Its maximum positioning error does not exceed $0.6 \mathrm{~m}$.Location error is less than $0.4 \%$.The algorithm reaches a high localization accuracy.

Table 3 :Indoor experiment” Location results (Unit: m)

\begin{tabular}{|c|c|c|c|c|c|}
\hline $\begin{array}{c}\text { Locatio } \\
\mathrm{n} \text { point }\end{array}$ & TCLA & WCLA & $\begin{array}{r}\text { Locati } \\
\text { on point }\end{array}$ & TCLA & WCLA \\
\hline 1 & 0.105 & 0.010 & 11 & 0.105 & 0.067 \\
\hline 2 & 0.154 & 0.073 & 12 & 0.075 & 0.041 \\
\hline 3 & 0.104 & 0.072 & 13 & 0.177 & 0.106 \\
\hline 4 & 0.127 & 0.080 & 14 & 0.089 & 0.094 \\
\hline 5 & 0.091 & 0.070 & 15 & 0.065 & 0.060 \\
\hline 6 & 0.153 & 0.110 & 16 & 0.108 & 0.076 \\
\hline 7 & 0.166 & 0.121 & 17 & 0.051 & 0.031 \\
\hline 8 & 0.084 & 0.058 & 18 & 0.098 & 0.036 \\
\hline 9 & 0.089 & 0.029 & 19 & 0.063 & 0.021 \\
\hline 10 & 0.133 & 0.071 & 20 & 0.106 & 0.069 \\
\hline
\end{tabular}

\section{Conclusion}

(1) This paper use wireless RSSI ranging principle.Use the trilateral centroid algorithm and WCLA respectively to conduct ranging and location.Through Matlab simulation, obtain positioning accuracy and relatederror information.Compare and analyse the two algorithms adaptive environment . Build an indoor location test system to do some tests. Further verify thesimulation results.

(2)Acoording toindoor location simulation analysis results, when reference nodes less than or equal three, trilateral centroid algorithm has a higher location accuracy. The calculation is simple ,while suitable for rapid calculation and transfer.With the number of reference nodes increasing, WCLA positioning error significantly reduced. Trilateral centroid algorithm Error has a poor stability. When the reference point ismore than three, we should use WCLA , which can significantly improve the precision and stability.

(3)Verifiedindoor experimental results analysis showed that the actual test positioning accuracy of WCLA is less than or equal $0.125 \mathrm{~m}$. The positioning error is less than $0.4 \%$.It is possible to obtain more accurate indoor robot location results.But through comparision between simulation and laboratory test,the accuracy of the algorithm measurement results will receive a certain extent affection. The control of measuement error need to befurther improved.

\section{References}

[1] HE T; HUANG C D; BLUM B M. Range-free localization schemes in large scale sensor networks[A].Proceedings of the Ninth Annual International Conference on Mobile Computing and Networking[C]. San Diego, United states, 2003. 81-95.

[2] LUTHY K A, E GRANT D, HENDERSON T C. Leveraging RSSI for robotic repair of disconnected wireless sensor networks [A].Proceedings of 2007 IEEE International Conference on Robotics and Automation[C].Rome, Italy,2007. 10-14.

[3] Kwon Oh-Heum,Song Ha-Joo,Park Sangjoon.The effects of stitching orders in patch-and-stitch WSNs localization algorithms[J].IEEE Transactions on Parallel and Distributed Systems, 2009, 20(9):1380-1391. 
nAutonomousDecentralizedSystem[C].Chengdu,Z000.36-39.

[5] LIONEL MN,Y NHAOL,YI CL,etal. LANDMARC: Indoor location Sensing sing Active RFID[A].ProceedingsoftheFirstIEEEInternationalConference[C],2003:407-415.

[6] Bulusu N, Heidemann J, Estrin D.GPS-less low cost outdoor localization for very small devices[J]. IEEE Personal Communications Magazine,2000,7(5): 28-34. 